\title{
Restricted Class of Colliding Einstein-Yang-Mills Plane Waves
}

\author{
Ozay Gurtug* and Mustafa Halilsoy \\ Department of Physics, Eastern Mediterranean University, \\ G. Magusa, north Cyprus, via Mersin 10, Turkey.
}

\begin{abstract}
By gauging an Abelian electromagnetic (em) solution through a non-Abelian transformation and in accordance with a theorem proved long time ago, we construct a simple class of colliding Einstein-Yang-Mills (EYM) plane waves. The solution is isometric to the Wu-Yang charged KerrNewman (KN) black hole and shares much of the properties satisfied by colliding Einstein-Maxwell (EM) plane waves. In the linear polarization limit with unit degenerate charge it reduces to the Bell-Szekeres (BS) solution for colliding em shock waves.
\end{abstract}

*Electronic address: ozay.gurtug@emu.edu.tr

†Electronic address: mustafa.halilsoy@emu.edu.tr 


\section{INTRODUCTION}

Starting with 1970s, through 80s, were the years in which the subject of colliding waves in general relativity attracted much interest. All known results were compiled later into a cataloque of solutions[1]. In later decades the interest in the subject continued with less momentum, concentrating more on the sophisticated fields such as dilaton, axion and torsion coupled to gravity and electromagnetic (em) fields. Formation of Cauchy horizon / singularity, and under what conditions the horizon remains stable dominated most discussions to date. To our knowledge the discussion has not been conclusive to the satisfaction of all yet. Our aim in this paper is not to contribute in this particular direction, but rather to point out a restricted class of colliding Yang-Mills (YM) waves which behaves almost em-like.

The motivating factor to consider such a problem anew relies on the recent attempts of Large Hadron Collisions ( $\mathrm{LHC}$ ) at $\mathrm{TeV}$ scale that maintain the prime agenda at CERN. As the protons (anti-protons) are boosted to almost the speed of light they behave more wave-like than particle-like whose collisions are reminiscent of waves colliding in general relativity. Since the inner/color structure of hadrons have constituent YM fields, collision of such waves deserves further investigation.

Within this context although there is a large collection of Einstein-Maxwell (EM) solutions available in the literature [1] [2], extension of the problem to the Einstein-Yang-Mills (EYM) remained ever open. We aim to contribute in this regard, at least partly, for the gauge group $S O(3)$ and pave the way for further solutions underlying various gauge groups. Our starting point is a Theorem proved long time ago by P. Yasskin [3] in connection with Yang-Mills (YM) fields in a curved spacetime. The method in Yasskin's Theorem is to start from an Abelian $U(1)$ em solution and map it through a non-Abelian transformation to the YM problem. In this process, naturally, the degenerate YM charges are defined from the

original em charge. By employing the Wu-Yang ansatz [4] for the YM fields in the trapped region of the Kerr-Newman (KN) black hole we construct solution that describes colliding EYM plane waves. In this process, rotation of the black hole transforms into the cross polarization of the colliding waves. In the linear polarization limit we recover the colliding em wave solution due to Bell and Szekeres (BS) [5]. More generally, any colliding EM metric/field can be shown to represent at the same time colliding EYM waves, in a restricted sense provided the YM field is defined according to the Yasskin's Theorem. This guaran- 
tees that the incoming YM fields are em-like plane waves so that they do not create extra currents. It can be anticipated that non-planar waves will induce their own sources through self-interaction which is the case that we avoid in the present study. Although YM fields is known to be a subject in the realm of quantum (chromodynamics), our treatment is entirely classical here. Stated otherwise, we adopt the viewpoint that anything that interacts with the classical gravity must itself behave classical.

Organization of the paper is as follows. In section II, we introduce YM fields in a KN black hole geometry. Colliding EYM plane waves follows in section III. Section IV, concentrates on the linear polarization of the waves. We complete the paper with conclusion in section V.

\section{KN BLACK HOLE AND YM FIELDS.}

The KN black hole solution is given by the line element

$$
d s^{2}=\frac{U^{2}}{\rho^{2}}\left(d t-\bar{a} \sin ^{2} \theta d \varphi\right)^{2}-\frac{\sin ^{2} \theta}{\rho^{2}}[F d \varphi-\bar{a} d t]^{2}-\frac{\rho^{2}}{U^{2}} d r^{2}-\rho^{2} d \theta^{2}
$$

where

$$
U^{2}=r^{2}-2 m r+\bar{a}^{2}+Q^{2}, \quad \rho^{2}=r^{2}+\bar{a}^{2} \cos ^{2} \theta, \quad F=r^{2}+\bar{a}^{2},
$$

in which $\bar{a}$ is the parameter of rotation and $Q$ is the em charge. By invoking Yasskin's Theorem [3] we extend this solution to represent a particular YM field as follows. A suitable YM gauge potential 1 -form $A^{i}=A_{\mu}^{i} d x^{\mu},(i=1,2,3)$ is chosen as

$$
A^{i}=\frac{1}{\rho^{2}} Q^{i} \cos \theta\left[\left(r^{2}+\bar{a}^{2}\right) d \varphi-\bar{a} d t\right] .
$$

Here the gauge charge $Q^{i}$ satisfies the constraint

$$
\gamma_{i j} Q^{i} Q^{j}=Q^{2}
$$

with the invariant group metric $\gamma_{i j}=\delta_{i j}$ and $Q$ is the charge of the KN black hole. The YM field 2-form is defined by $F^{i}=\frac{1}{2} F_{\mu \nu}^{i} d x^{\mu} \wedge d x^{\nu}$, where $\wedge$ stands for the wedge product and 


$$
F_{\mu \nu}^{i}=\partial_{\mu} A_{\nu}^{i}-\partial_{\nu} A_{\mu}^{i}+\frac{1}{2 Q} \epsilon_{j k}^{i} A_{\mu}^{j} A_{\nu}^{k},
$$

in which $\epsilon_{j k}^{i}$ is the structure constant for the group. In the $S O(3)$ basis, $T^{i}(i=1,2,3)$ are given by

$$
T^{1}=\left(\begin{array}{ccc}
0 & 0 & 0 \\
0 & 0 & -1 \\
0 & 1 & 0
\end{array}\right), \quad T^{2}=\left(\begin{array}{ccc}
0 & 0 & 1 \\
0 & 0 & 0 \\
-1 & 0 & 0
\end{array}\right), \quad T^{3}=\left(\begin{array}{ccc}
0 & -1 & 0 \\
1 & 0 & 0 \\
0 & 0 & 0
\end{array}\right),
$$

and the YM potential 1-form has the representation

$$
A=A_{\mu}^{i} T^{i} d x^{\mu} .
$$

For the particular choice $\left(Q^{3}=Q \neq 0=Q^{1}=Q^{2}\right)$ we have

$$
\begin{aligned}
A_{\varphi}^{3} & =\frac{Q \cos \theta}{\rho^{2}}\left(r^{2}+\bar{a}^{2}\right), \\
A_{t}^{3} & =-\frac{\bar{a} Q \cos \theta}{\rho^{2}},
\end{aligned}
$$

which has both electric and magnetic components. If $\bar{a}=0$, i.e. for the Reissner-Nordstrom black hole, we have a pure magnetic field. Now we apply a gauge transformation on $A_{\mu}$ through

$$
A_{\mu} \rightarrow \widetilde{A}_{\mu}=G A_{\mu} G^{-1}-Q\left(\partial_{\mu} G\right) G^{-1},
$$

where the gauge transformation matrix $G$ is

$$
G=\left(\begin{array}{ccc}
\sin \varphi & \cos \varphi \cos \theta & \cos \varphi \sin \theta \\
-\cos \varphi & \sin \varphi \cos \theta & \sin \varphi \sin \theta \\
0 & -\sin \theta & \cos \theta
\end{array}\right)
$$

We obtain the components of the new gauge potential 1-forms (after suppressing the tilde over $A_{\mu}$ ) as 


$$
\begin{aligned}
& A^{1}=\frac{Q}{2 \rho^{2}} \sin 2 \theta \cos \varphi\left[\left(r^{2}+\bar{a}^{2}\right) d \varphi-\bar{a} d t\right]+Q \sin \varphi d \theta, \\
& A^{2}=\frac{Q}{2 \rho^{2}} \sin 2 \theta \sin \varphi\left[\left(r^{2}+\bar{a}^{2}\right) d \varphi-\bar{a} d t\right]-Q \cos \varphi d \theta, \\
& A^{3}=-\frac{Q}{\rho^{2}}\left(r^{2} \sin ^{2} \theta d \varphi+\bar{a} \cos ^{2} \theta d t\right) .
\end{aligned}
$$

In the next section we shall transform these potentials and metric (1) into the colliding wave spacetime and interpret it as a solution to the colliding EYM problem.

\section{COLLIDING EYM PLANE WAVES.}

The metric function $U^{2}=r^{2}-2 m r+\bar{a}^{2}+Q^{2}=0$, in Eq.(2) has two roots, $r=r_{+}$(the outer horizon) and , $r=r_{-}$(the inner horizon). By the particular choice of parameters it is possible to make $r_{+}=r_{-}$, which is called the extremal case, however, we shall consider here only the case $r_{+}>r_{-} \neq 0$. It can easily be seen that for $r_{-}<r<r_{+}, U^{2}<0$, which makes the spacetime to admit two spacelike Killing vectors apt for colliding waves. For simplicity we choose the mass, $m=1$ and apply the following transformation

$$
\begin{gathered}
r=1+\alpha \tau, \quad \sigma=\cos \theta, \quad t=\alpha x, \quad y=\varphi, \\
\left(\alpha=\sqrt{1-\bar{a}^{2}-Q^{2}}=\frac{1}{p}\right)
\end{gathered}
$$

to the line element (1). After an overall scaling of the metric we obtain

$$
d s^{2}=X\left(\frac{d \tau^{2}}{\Delta}-\frac{d \sigma^{2}}{\delta}\right)-X^{-1}\left(R d x^{2}+E d y^{2}-2 G d x d y\right)
$$

where

$$
\begin{aligned}
& X=(p+\tau)^{2}+a_{0}^{2} \sigma^{2}, \quad R=\Delta+a_{0}^{2} \delta, \\
& E=\Delta A^{2}+\delta B^{2}, \quad G=\Delta A+a_{0} \delta B, \\
& A=a_{0} \delta, \quad B=(p+\tau)^{2}+a_{0}^{2}, \\
& \Delta=1-\tau^{2}, \quad \delta=1-\sigma^{2},
\end{aligned}
$$

in which 


$$
\tau=\sin (a u \theta(u)+b v \theta(v)), \quad \sigma=\sin (a u \theta(u)-b v \theta(v)),
$$

are the new coordinates in terms of the null coordinates $(u, v)$ and $(a, b)$ are arbitrary constants. We note also that $a_{0}=\bar{a} p$. By introducing another constant $q=Q p$, we can check that the constraint condition

$$
p^{2}-a_{0}^{2}-q^{2}=1
$$

holds. The YM potential 1-forms are now

$$
\begin{aligned}
& A^{1}=\frac{Q \sigma \sqrt{\delta}}{X} \cos y\left(B d y-a_{0} d x\right)-Q \sin y \frac{d \sigma}{\sqrt{\delta}} \\
& A^{2}=\frac{Q \sigma \sqrt{\delta}}{X} \sin y\left(B d y-a_{0} d x\right)+Q \cos y \frac{d \sigma}{\sqrt{\delta}} \\
& A^{3}=\frac{Q \sigma^{2}}{X}\left(B d y-a_{0} d x\right)-d y .
\end{aligned}
$$

We note that in the null coordinates $(u, v)$ we have

$$
\begin{aligned}
& d \sigma=-\sqrt{\delta}(a \theta(u) d u-b \theta(v) d v) \\
& d \tau=\sqrt{\Delta}(a \theta(u) d u+b \theta(v) d v)
\end{aligned}
$$

in which $\theta(u)$ and $\theta(v)$ are the unit step functions introduced as the requirement of the collision problem. Let us note that insertion of the step functions must be checked critically to ensure the absence of any redundant current sheets. In most cases such an insertion fails to work but here, due to its em analogy it does work. The YM field 2-form $F^{i}$ are given by.

$$
F^{i}=Q \sqrt{\delta}\left(\cos y, \sin y, \frac{\sigma}{\sqrt{\delta}}\right) F
$$

where the 2 -form $F$ is

$$
F=\frac{1}{X^{2}}\left[\left((p+\tau)^{2}-a_{0}^{2} \sigma^{2}\right) d \sigma-2 \sigma(p+\tau) d \tau\right] \wedge\left(B d y-a_{0} d x\right),
$$

while its dual takes the form

$$
{ }^{*} F=\frac{1}{X^{2}}\left[\left((p+\tau)^{2}-a_{0}^{2} \sigma^{2}\right) d \tau \wedge\left(d x-a_{0} \delta d y\right)-2 a_{0} \sigma(p+\tau) d \sigma \wedge\left(B d y-a_{0} d x\right)\right] .
$$


Without much difficulty, which is more transparent in the $(\tau, \sigma, x, y)$, compared with the $(u, v, x, y)$ coordinates, one can show that the integrability equations

$$
d F+A \wedge F=0
$$

and the YM equations

$$
d^{*} F+A \wedge^{*} F=0
$$

are all satisfied. This solves the problem of colliding EYM plane waves where the YM field is obtained from the theorem of Yasskin while the metric is the inner horizon region of the KN geometry explored first within the context of EM by Chandrasekhar and Xanthopoulos [6]. For a detailed analysis of this spacetime we refer to [6].

\section{THE LINEAR POLARIZATION LIMIT.}

In this section we consider the metric obtained in the previous section and set $a_{0}=0$ to make waves linearly polarized. In the null coordinates after an overall scaling the line element takes the form

$$
d s^{2}=\Sigma^{2}\left(2 d u d v-\delta d y^{2}\right)-\frac{\Delta}{\Sigma^{2}} d x^{2}
$$

where

$$
\Sigma=1+\alpha \tau, \quad \Delta=1-\tau^{2}, \quad \delta=1-\sigma^{2}, \quad \alpha=\sqrt{1-Q^{2}},
$$

while the $S O(3)$ valued gauge potential 1-forms are

$$
\begin{aligned}
& A^{1}=Q[(\sigma \sqrt{\delta} \cos y) d y-(\sin y)(a \theta(u) d u-b \theta(v) d v)] \\
& A^{2}=Q[(\sigma \sqrt{\delta} \sin y) d y+(\cos y)(a \theta(u) d u-b \theta(v) d v)] \\
& A^{3}=-Q \delta d y
\end{aligned}
$$

The YM field 2-form $F^{i}$ and ${ }^{*} F^{i}$ can be expressed as 


$$
\begin{aligned}
F^{i} & =Q \delta\left(\cos y, \sin y, \frac{\sigma}{\sqrt{\delta}}\right)[a \theta(u) d u-b \theta(v) d v] \wedge d y \\
{ }^{*} F^{i} & =\frac{Q \sqrt{\Delta \delta}}{(\Sigma)^{2}}\left(\cos y, \sin y, \frac{\sigma}{\sqrt{\delta}}\right)[a \theta(u) d u+b \theta(v) d v] \wedge d x
\end{aligned}
$$

In the null tetrad basis 1 -forms $(l, n, m, \bar{m})$ of Newman and Penrose

$$
\begin{aligned}
l & =\Sigma d u \\
n & =\Sigma d v \\
m+\bar{m} & =\sqrt{2} \Sigma \sqrt{\delta} d y, \\
m-\bar{m} & =\sqrt{2} i \frac{\sqrt{\Delta}}{\Sigma} d x,
\end{aligned}
$$

the energy-momentum tensor $T_{\mu \nu}$ becomes,

$$
4 \pi T_{\mu \nu}=\frac{Q^{2}}{\Sigma^{4}}\left[a^{2} \theta(u) l_{\mu} l_{\nu}+b^{2} \theta(v) n_{\mu} n_{\nu}+a b \theta(u) \theta(v)\left(m_{\mu} m_{\nu}+\bar{m}_{\mu} \bar{m}_{\nu}\right)\right] .
$$

Prior to the collision the incoming, coupled EYM plane waves are obtained by setting $v<0$ $(u<0)$ in Eq.(24). For $u<0$ and $v<0$ we have a flat space in which the YM field vanishes. For $u>0$ and $v>0$, the Weyl scalars $\Psi_{2}, \Psi_{0}$ and $\Psi_{4}$ are all regular as can be checked from

$$
\begin{aligned}
& \Psi_{2}=\frac{\alpha(\alpha+\tau)}{\Sigma^{4} a b \theta(u) \theta(v),} \\
& \Psi_{4}=-\frac{a \delta(u)[\alpha+\sin (b v \theta(v))]}{\cos (b v \theta(v))[1+\alpha \sin (b v \theta(v))]}+3 a^{2} \theta(u) \frac{\alpha(\alpha+\tau)}{\Sigma^{4}} \\
& \Psi_{0}=-\frac{b \delta(v)[\alpha+\sin (a u \theta(u))]}{\cos (a u \theta(u))[1+\alpha \sin (a u \theta(u))]}+3 b^{2} \theta(v) \frac{\alpha(\alpha+\tau)}{\Sigma^{4}}
\end{aligned}
$$

satisfying the type-D condition $\Psi_{0} \Psi_{4}=9 \Psi_{2}^{2}$. The invariants $I=2\left(\Psi_{0} \Psi_{4}+3 \Psi_{2}^{2}\right)$ and $J=$ $6 \Psi_{2}\left(\Psi_{0} \Psi_{4}-\Psi_{2}^{2}\right)$ are both regular as they should be in the inside of the collision region. On the boudaries $u=0, b v=\pi / 2(v=0, a u=\pi / 2)$, however, there are null singularities which are also present in the problem of colliding em shock waves[5]. The pathology involved ( if any) on the hypersurfaces $\tau=1$ and $\sigma= \pm 1$ has been discussed extensively in the literature of colliding waves ( see Ref.[1] and references therein). An analytic extension beyond the horizon $(\tau=1)$, albeit it is a non - unique process, reveals the geodesics completeness and other issues [7]. 
The metric (23) has well known limits. For $Q=0(\alpha=1)$ the YM field vanishes and one recovers the colliding gravitational waves locally isometric to the Schwarzschild interior[1]. For $Q=1(\alpha=0)$ we have the case of colliding em waves [5]. This implies that the BellSzekeres metric can be interpreted at the same time to represent colliding YM plane waves for a unit charge $Q=1$.

The Wu-Yang ansatz solution[4] for the static YM fields has the gauge potential

$$
A^{i}=-\frac{Q}{r^{2}} \epsilon_{j k}^{i} x^{k} d x^{j}
$$

where $x^{i}$ stands for the Cartesian coordinates and $Q=Q^{3} \neq 0$, is the only non-zero gauge charge. By the substitution, $x^{1}=r \sin \theta \cos \varphi, x^{2}=r \sin \theta \sin \varphi$ and $x^{3}=r \cos \theta$, followed by $\cos \theta=\sin (a u \theta(u)-b v \theta(v))$ and $y=\varphi$, one obtains potential 1-forms ( Eq.(25)). The YM potentials in Eq.(25) just corresponds to the curved space generalization of the Wu-Yang ansatz solution[4].

\section{CONCLUSION.}

Customarily YM fields arise as part of a quantum theory inside nuclei. It carries its own gauge charge and self-interacts with itself. These properties are fundamentally different from em waves which correspond to the classical equivalence of photons. In this paper we treat YM waves along with gravitational waves as classical and consider their collision problem in a restricted form. This is not to be compared with a Feynman diagram of quantum chromodynamics. The highly non-linear YM waves acts in this simple picture parallel to gravitational waves to distort spacetime upon interaction. The example which we present here is a case that forms Cauchy horizon ( of Reissner-Nordstrom and KN types) instead of a singularity. Yasskin's theorem and the Wu-Yang ansatz aided in obtaining this simple class. Different combinations/collisions which employ the full non-linearity and non-Abelian character may change this picture completely. This, however, remains as challenging as ever.

[1] J.B. Griffiths. Colliding Plane Waves in General Relativity, Oxford University Press, Oxford. (1991).

[2] M. Halilsoy. J. Math. Phys. (NY), 31, 2694-2698, (1990). 
[3] P.B. Yasskin, Phys. Rev. D12, 2212-2217, (1975).

[4] T.T. Wu and C.N. Yang, in Properties of Matter Under Unusual Conditions, edited by H. Mark and S. Fernbach ( Interscience, New York, 1969).

[5] P. Bell and P. Szekeres. Gen. Rel. and Grav. 5, 275. (1974).

[6] S. Chandrasekhar and B.C. Xanthopoulos. Proc. R. Soc. London, A414, 1. (1987).

[7] O. Gurtug and M. Halilsoy, Int. Journal Mod. Phys. A. 24, 3171. (2009). 